\title{
Aplicación Clínica del lonómero Vítreo de restauración con Amalgama Dental como alternativa rehabilitadora en pie- zas dentarias con gran destrucción de paredes cavitarias.
}

Clinical application of glass ionomer with dental amalgam as alternative treatment of

teeth with extensive coronal destruction.

Fecha de Recepción

06 de septiembre de 2012
Aplicação clínica de ionômero de vidro com amálgama em dentes com grande destruição das paredes da cavidade.

Aceptado para su publicación

05 de diciembre de 2012
María Natalia Mandri

Auxiliar Docente $I^{\circ}$ Categoría. Cátedra Preclínica de Operatoria Dental.

María Eugenia Zamudio Profesora Titular. Cátedra de Biomateriales.

Alicia Aguirre Grabre

Profesora Titular. Cátedra de Preclínica de Operatoria Dental.

Lugar de Trabajo Facultad de Odontología UNNE

Av. Libertad 5450

Corrientes, Argentina.

\section{Resumen}

El propósito del estudio fue evaluar a los 3 y 6 meses, la respuesta clínica de diferentes cementos de lonómero Vítreo utilizados con la Amalgama Dental como restauración permanente en dientes posteriores con gran destrucción de paredes cavitarias. Se seleccionaron 20 piezas dentarias, de acuerdo a los criterios de inclusión establecidos, en las cuales se realizó el tratamiento restaurador correspondiente, distribuyéndose aleatoriamente en los siguientes grupos: Grupo I, lonomero Vítreo modificado con Resina/Amalgama y Grupo 2, lonomero Vítreo convencional/Amalgama. La evaluación clínica de las restauraciones se realizó acorde a los criterios generales Ryge/USPHS modificado. Los resultados obtenidos en las evaluaciones a los 3 y 6 meses demostraron que las piezas dentarias restauradas del grupo I presentaron un mayor porcentaje de éxito que el grupo 2 , determinándose además la ausencia de caries secundaria en la totalidad de los casos examinados.

\section{Pallabras claves}

Biomateriales, desempeño clínico, dientes permanentes, restauraciones.

\section{Summary}

The purpose of the study was to assess at 3 and 6 months, the clinical response of different Glass lonomer Cements used with Dental Amalgam as permanent restoration of posterior teeth with cavity walls great destruction. 20 
teeth were selected according to inclusion criteria established, in which restorative treatment was performed, randomly distributed into the following groups: group I, Resin-modified Glass lonomer/Amalgam and Group 2, Conventional Glass lonomer/Amalgam. Clinical evaluation of the restorations was performed according to modified USPHS/Ryge criteria. The results of the evaluations at 3 and 6 months showed that the restored teeth in group I had a higher success rate than group 2 , also determined the absence of secondary caries in the all the cases examined.

\section{Key words}

Biomaterials, clinical performance, permanent teeth, restorations.

\section{Resumo}

O objetivo do estudo foi avaliar a 3 e 6 meses, a resposta clínica de cimentos de lonômero de Vidro diferentes utilizados com Amálgama Dental como restauração permanente dos dentes posteriores com grande destruição de paredes duplas de 20 dentes foram selecionados de acordo com os critérios inclusão estabelecido, em que o tratamento restaurador foi adequado, distribuídos aleatoriamente nos seguintes grupos: Grupo I, modificado por Resina de lonômero de Vidro/Amálgama e Grupo 2, lonômero de Vidro Convencional/Amálgama. A avaliação clínica das restaurações foi realizada de acordo com os critérios gerais Ryge/USPHS modificado. Os resultados das avaliações de 3 e 6 meses mostraram que os dentes restaurados do grupo I tinham uma taxa de sucesso mais elevada do que o grupo 2 , também determinada na ausência de cárie no todos os casos examinados.

\section{Palavras chaves}

Biomateriais, desempenho clínico, os dentes permanentes, restaurações.

\section{Introducción}

La Amalgama ha sido, sin lugar a duda, el material restaurador más exhaustivamente investigado y utilizado durante muchos años'. Entre sus propiedades se destacan: resistencia, bajo costo, longevidad ${ }^{2,3}$, durabilidad y facilidad de manipulación. Por estas razones, se convirtió una valiosa opción en el tratamiento odontológico restaurador, especialmente en cavidades clase Iy II, áreas donde las cargas masticatorias son mayores y el factor estético no adquiere fundamental relevancia ${ }^{4,5}$.

En el último tiempo, sin embargo, se ha observado como rutina el reemplazo de las restauraciones de Amalgama frente al más mínimo defecto o a la posibilidad de fracaso de las mismas, especialmente en piezas dentarias con gran destrucción de paredes cavitarias ${ }^{6}$. En este sentido, numerosos estudios se han llevado a cabo para determinar las razones de reemplazo de restauraciones de Amalgama ${ }^{7}$. En estas investigaciones las principales causas de reemplazo reportadas han sido la caries secundaria, la fractura de la restauración y la fractura marginal ${ }^{8,9}$. La razón de la mayor parte de estos inconvenientes se debe a su incapacidad para unirse adhesivamente a los sustratos dentales.

Los cementos de lonómero Vítreo son frecuentemente conocidos como materiales biomiméticos, debido a sus propiedades mecánicas similares a la dentina. Esta característica, junto con los importantes beneficios de liberación de flúor y adhesión a la estructura dentaria, los hace un material ideal en diversas situaciones restaurativas $^{10}$. El mecanismo de unión es variable según se trate de un cemento lonómero Vítreo convencional o uno modificado con resina" y permite varios beneficios entre los cuales pueden mencionarse: el sellado de la cavidad, la protección del tejido pulpar, la prevención de microfiltración y el inicio de caries secundaria, y el refuerzo, en alguna medida, de la estructura dentaria remanente debido a la integración tejido dentariomaterial restaurador.

Una importante desventaja de los lonómeros Vítreos es que son sensibles a deshidratación temprana en el proceso de asentamiento, particularmente en el caso de los convencionales, y son materiales quebradizos, por ende no apropiados como material restaurador final para uso en áreas de alto stress, donde deberían ser protegidos con Resina Compuesta o Amalgama ${ }^{12-14}$. En virtud de lo expuesto, la utilización de lonómero Vítreo con Amalgama en el tratamiento de molares y premolares permanentes con lesiones cariosas y gran destrucción de las paredes cavitarias, resultaría en un aumento de la resistencia de unión de la Amalgama a la estructura dentaria. La unión 
mecanoquímica y la liberación de fluoruro sostenida debido al lonómero Vítreo aumentaría la retención de las restauraciones con amalgama, y se espera que sea beneficioso para aumentar la longevidad de la restauración debido a la disminución de la filtración marginal, de la fractura del diente y la incidencia de caries recurrentes.

El propósito del estudio fue evaluar a los 3 y 6 meses, la respuesta clínica de diferentes cementos de lonómero Vítreo utilizados con la Amalgama dental como restauración permanente en piezas dentarias posteriores con gran destrucción de paredes cavitarias.

\section{Materiales y Métodos}

Previa explicación del proyecto a realizarse se solicitó el consentimiento informado de los participantes del estudio, se seleccionaron 20 piezas dentarias en pacientes que concurrieron para atención clínica al Hospital de la Facultad de Odontología de la U.N.N.E, de acuerdo a los siguientes criterios de inclusión:

- Pacientes sanos

- Pacientes con una o más piezas dentarias con lesiones cariosas de superficies múltiples, pudiendo haber antecedentes de sintomatología dolorosa provocada, de corta duración.

- Piezas dentarias con ausencia de sintomatología clínica, que sugieran la posibilidad de la presencia de pulpa irreversiblemente inflamada como ser: dolor espontáneo, presencia de fístula, edema, movilidad y dolor a la percusión.

- Piezas dentarias con ausencia de lesiones radiográficas apicales.

Seleccionado el paciente, el día 0 o inicio del estudio se realizó un examen clínico y toma de radiografías periapicales para diagnóstico.

A continuación, las piezas dentarias elegidas fueron asignadas aleatoriamente a cada uno de los siguientes grupos experimentales:

- Grupo I: Conformado por 10 piezas dentarias, a las cuales se utilizó lonómero Vítreo modificado con resina (Vitremer ${ }^{B} 3 \mathrm{M}$ St Paul MN, USA)/Amalgama.

- Grupo 2: Conformado por 10 piezas dentarias, a las cuales se colocó lonómero Vítreo convencional (Ketac ® Molar de ESPE)/Amalgama.

Para la restauración se procedió de acuerdo a la siguiente técnica: previa administración de anestesia local y aislamiento absoluto de la pieza dentaria se realizó la apertura de la cavidad con el uso de instrumental rotatorio a alta velocidad seguido de la utilización de instrumental de mano con el fin de eliminar completamente el tejido cariado de la cavidad. Se lavó la cavidad con agua destilada y se secó con aire, colocándose luego, sobre la dentina expuesta, el material a evaluarse (de acuerdo a las indicaciones del fabricante).La evaluación de las restauraciones se realizó de acuerdo a los criterios clínicos generales Ryge/ USPHS modificado ${ }^{15-17}$ (Tabla I).

\section{Resultados}

El tratamiento de restauración fue realizado en 20 piezas dentarias cuyas evaluaciones clínicas, se distribuyeron de la manera representada en la Tabla II.

En ambas evaluaciones, la forma anatómica y el contorno axial de las restauraciones resultaron clínicamente ideales en la totalidad de los casos del grupo I, observándose similar resultado sólo en un $80 \%$ de los casos del grupo 2 (Tabla III y IV). En la Tabla $V$ se puede observar la integridad marginal de las restauraciones en los distintos grupos a lo largo del diseño experimental, expresado en porcentaje y número de piezas dentarias. En las evaluaciones realizadas a los 3 y 6 meses, las piezas dentarias restauradas con lonómero Vítreo modificado con Resina/Amalgama presentaron un mayor porcentaje de éxito (Alfa (A) - 100\%) que el grupo restaurado con lonómero Vítreo Convencional/ Amalgama.

La Tabla VI demuestra la ausencia de caries secundaria en la totalidad de los casos de ambos grupos experimentales.

\section{Discusión}

Desde el momento de su introducción, la Amalgama ha demostrado ser un material restaurador permanente con una buena longevidad. Sin embargo, la falta de adhesividad de las estructuras dentarias puede dar lugar a situaciones, que llevan al fracaso de la restauración a largo plazo, como la fractura dentaria y la fractura de la restauración. En este sentido, la resistencia a la fractura de los dientes restaurados con la técnica de Amalgama usando lonómero Vítreo restaurador como adhesivo ha demostrado ser mayor que en los 
Tabla I: Criterios clínicos generales Ryge/USPHS modificado.

\begin{tabular}{|c|c|c|c|}
\hline $\begin{array}{l}\text { Características } \\
\text { Clínicas }\end{array}$ & $\begin{array}{c}\text { ALFA (A) } \\
\text { Clínicamente ideal }\end{array}$ & $\begin{array}{l}\text { BRAVO (B) } \\
\text { Clínicamente } \\
\text { aceptable }\end{array}$ & $\begin{array}{l}\text { CHARLIE (C) } \\
\text { Clínicamente } \\
\text { inaceptable }\end{array}$ \\
\hline Forma Anatómica & $\begin{array}{l}\text { Continuidad de la restau- } \\
\text { ración con la estructura } \\
\text { adyacente. No hay pérdi- } \\
\text { da de sustancia. }\end{array}$ & $\begin{array}{l}\text { Eliminación parcial de la } \\
\text { restauración con pérdida } \\
\text { de sustancia. }\end{array}$ & $\begin{array}{l}\text { Eliminación total de la } \\
\text { restauración. }\end{array}$ \\
\hline Contorno Axial & $\begin{array}{l}\text { El contorno general de la } \\
\text { restauración sigue el con- } \\
\text { torno del diente. }\end{array}$ & $\begin{array}{l}\text { El contorno general de la } \\
\text { restauración no sigue el } \\
\text { contorno del diente (Sub- } \\
\text { contorno) }\end{array}$ & Sobrecontorno. \\
\hline Integridad Marginal & $\begin{array}{l}\text { El explorador no se traba } \\
\text { o se traba en una direc- } \\
\text { ción cuando se examina } \\
\text { a través de la interfase } \\
\text { diente/restauración. }\end{array}$ & $\begin{array}{l}\text { El explorador cae en una } \\
\text { brecha cuando se exami- } \\
\text { na la interfase diente/ res- } \\
\text { tauración. }\end{array}$ & $\begin{array}{l}\text { La dentina o base cavita- } \\
\text { ria están expuestas a lo } \\
\text { largo del margen. }\end{array}$ \\
\hline Caries Secundaria & Ausencia de caries. & & $\begin{array}{l}\text { Caries asociada a los már- } \\
\text { genes de la restauración. }\end{array}$ \\
\hline
\end{tabular}

Tabla II: Distribución del número de piezas dentarias examinadas en el primer y segundo control clínico.

\begin{tabular}{|l|c|c|}
\hline Grupos Experimentales & $\begin{array}{c}\text { Evaluación I } \\
(3 \text { meses })\end{array}$ & $\begin{array}{c}\text { Evaluación II } \\
(6 \text { meses })\end{array}$ \\
\hline $\begin{array}{l}\text { Grupo I: lonomero Vitreo modificado con Resi- } \\
\text { na (Vitremer ® 3M St Paul MN, USA)/Amalgama }\end{array}$ & 10 & 10 \\
\hline $\begin{array}{l}\text { Grupo 2: Ionomero Vítreo Convencional (Ketac } \\
\text { ® Molar de ESPE)/Amalgama. }\end{array}$ & 10 & 10 \\
\hline
\end{tabular}

Tabla III: Forma anatómica observada clínicamente durante la primera y segunda evaluación, expresada en $\%$ y $\mathrm{N}^{\circ}$ ( ) de piezas dentarias observadas en cada categoría.

\begin{tabular}{|c|c|c|c|c|c|c|}
\hline \multirow[t]{2}{*}{$\begin{array}{c}\text { Grupos } \\
\text { Experimentales }\end{array}$} & \multicolumn{3}{|c|}{$\begin{array}{c}\text { Evaluación I } \\
\text { (3 meses) }\end{array}$} & \multicolumn{3}{|c|}{$\begin{array}{c}\text { Evaluación II } \\
\text { (6 meses) }\end{array}$} \\
\hline & $A^{*}$ & $\mathrm{~B}^{*}$ & $\mathrm{CH}^{*}$ & $A^{*}$ & $\mathrm{~B}^{*}$ & $\mathrm{CH}^{*}$ \\
\hline $\begin{array}{l}\text { Grupo I: lonomero Vítreo } \\
\text { modificado con Resina (Vi- } \\
\text { tremer } \circledast 3 M \text { St Paul MN, } \\
\text { USA)/restauración }\end{array}$ & $\begin{array}{r}100 \% \\
(10)\end{array}$ & $\begin{array}{l}0 \% \\
(0)\end{array}$ & $\begin{array}{l}0 \% \\
(0)\end{array}$ & $\begin{array}{r}100 \% \\
(10)\end{array}$ & $\begin{array}{l}0 \% \\
(0)\end{array}$ & $\begin{array}{l}0 \% \\
(0)\end{array}$ \\
\hline $\begin{array}{l}\text { Grupo 2: lonomeroVitreo } \\
\text { Convencional (Ketac } ® \text { Mo- } \\
\text { lar de ESPE)/Amalgama. }\end{array}$ & $\begin{array}{c}80 \% \\
(8)\end{array}$ & $\begin{array}{r}20 \% \\
(2)\end{array}$ & $\begin{array}{l}0 \% \\
(0)\end{array}$ & $\begin{array}{c}80 \% \\
(8)\end{array}$ & $\begin{array}{c}20 \% \\
(2)\end{array}$ & $\begin{array}{l}0 \% \\
(0)\end{array}$ \\
\hline
\end{tabular}

* (A) Alfa $=$ clínicamente ideal * (B) Bravo= clínicamente aceptable * (C) Charlie= clínicamente inaceptable 
Tabla IV: Contorno axial observado clínicamente durante la primera y segunda evaluación, expresada en $\%$ y $\mathrm{N}^{\circ}$ ( ) de piezas dentarias observadas en cada categoría.

\begin{tabular}{|c|c|c|c|c|c|c|}
\hline \multirow{2}{*}{$\begin{array}{c}\text { Grupos } \\
\text { Experimentales }\end{array}$} & \multicolumn{3}{|c|}{$\begin{array}{c}\text { Evaluación I } \\
\text { (3 meses) }\end{array}$} & \multicolumn{3}{|c|}{$\begin{array}{c}\text { Evaluación II } \\
\text { (6 meses) }\end{array}$} \\
\hline & $A^{*}$ & $\mathrm{~B}^{*}$ & $\mathrm{CH}^{*}$ & $A^{*}$ & $\mathrm{~B}^{*}$ & $\mathrm{CH}^{*}$ \\
\hline $\begin{array}{l}\text { Grupo I: lonomero Vitreo } \\
\text { modificado con Resina (Vi- } \\
\text { tremer } \AA 3 M \text { St Paul MN, } \\
\text { USA)/Amalgama. }\end{array}$ & $\begin{array}{l}100 \% \\
(10)\end{array}$ & $\begin{array}{l}0 \% \\
(0)\end{array}$ & $\begin{array}{l}0 \% \\
(0)\end{array}$ & $\begin{array}{c}100 \% \\
(10)\end{array}$ & $\begin{array}{l}0 \% \\
(0)\end{array}$ & $\begin{array}{l}0 \% \\
(0)\end{array}$ \\
\hline $\begin{array}{l}\text { Grupo 2: lonomero Vítreo } \\
\text { convencional (Ketac } ® \text { Mo- } \\
\text { lar de ESPE)/Amalgama. }\end{array}$ & $\begin{array}{c}80 \% \\
(8)\end{array}$ & $\begin{array}{c}20 \% \\
(2)\end{array}$ & $\begin{array}{l}0 \% \\
(0)\end{array}$ & $\begin{array}{c}80 \% \\
(8)\end{array}$ & $\begin{array}{c}20 \% \\
(2)\end{array}$ & $\begin{array}{l}0 \% \\
(0)\end{array}$ \\
\hline
\end{tabular}

* (A) Alfa= clínicamente ideal. * (B) Bravo= clínicamente aceptable. * (C) Charlie= clínicamente inaceptable.

Tabla V: Integridad marginal observada clínicamente durante la primera y segunda evaluación, expresada en $\%$ y $\mathrm{N}^{\circ}$ ( ) de piezas dentarias observadas en cada categoría.

\begin{tabular}{|c|c|c|c|c|c|c|}
\hline \multirow{2}{*}{$\begin{array}{c}\text { Grupos } \\
\text { Experimentales }\end{array}$} & \multicolumn{3}{|c|}{$\begin{array}{c}\text { Evaluación I } \\
\text { (3 meses) }\end{array}$} & \multicolumn{3}{|c|}{$\begin{array}{c}\text { Evaluación II } \\
\text { (6 meses) }\end{array}$} \\
\hline & $A^{*}$ & $B^{*}$ & $\mathrm{CH}^{*}$ & $A^{*}$ & $\mathrm{~B}^{*}$ & $\mathrm{CH}^{*}$ \\
\hline $\begin{array}{l}\text { Grupo I: lonomero Vítreo } \\
\text { modificado con Resina (Vi- } \\
\text { tremer } \AA 3 M \text { St Paul MN, } \\
\text { USA)/Amalgama. }\end{array}$ & $\begin{array}{c}100 \% \\
(10)\end{array}$ & $\begin{array}{l}0 \% \\
(0)\end{array}$ & $\begin{array}{l}0 \% \\
(0)\end{array}$ & $\begin{array}{c}100 \% \\
(10)\end{array}$ & $\begin{array}{l}0 \% \\
(0)\end{array}$ & $\begin{array}{l}0 \% \\
(0)\end{array}$ \\
\hline $\begin{array}{l}\text { Grupo 2: lonomero Vitreo } \\
\text { convencional (Ketac } \AA \text { Mo- } \\
\text { lar de ESPE)/Amalgama. }\end{array}$ & $\begin{array}{c}80 \% \\
(8)\end{array}$ & $\begin{array}{c}20 \% \\
(2)\end{array}$ & $\begin{array}{l}0 \% \\
(0)\end{array}$ & $\begin{array}{c}80 \% \\
(8)\end{array}$ & $\begin{array}{c}20 \% \\
(2)\end{array}$ & $\begin{array}{l}0 \% \\
(0)\end{array}$ \\
\hline
\end{tabular}

* (A) Alfa = clínicamente ideal. * (B) Bravo= clínicamente aceptable. * (C) Charlie= clínicamente inaceptable

Tabla VI: Ausencia/presencia de caries secundaria observada durante la primera y segunda evaluación, expresada en \% y $\mathrm{N}^{\circ}$ ( ) de piezas dentarias observadas en cada categoría.

\begin{tabular}{|c|c|c|c|c|}
\hline \multirow{2}{*}{$\begin{array}{c}\text { Grupos } \\
\text { Experimentales }\end{array}$} & \multicolumn{2}{|c|}{$\begin{array}{c}\text { Evaluación I } \\
\text { (3 meses) }\end{array}$} & \multicolumn{2}{|c|}{$\begin{array}{c}\text { Evaluación II } \\
\text { (6 meses) }\end{array}$} \\
\hline & $A^{*}$ & $\mathrm{CH}^{*}$ & $A^{*}$ & $\mathrm{CH}^{*}$ \\
\hline $\begin{array}{l}\text { Grupo I: lonomero Vítreo } \\
\text { modificado con Resina (Vi- } \\
\text { tremer } \AA 3 M \text { St Paul MN, } \\
\text { USA)/Amalgama. }\end{array}$ & $\begin{array}{c}100 \% \\
(10)\end{array}$ & $\begin{array}{l}0 \% \\
(0)\end{array}$ & $\begin{array}{l}100 \% \\
(10)\end{array}$ & $\begin{array}{l}0 \% \\
(0)\end{array}$ \\
\hline $\begin{array}{l}\text { Grupo 2: lonomero Vitreo } \\
\text { convencional (Ketac } ₫ \text { Mo- } \\
\text { lar de ESPE)/Amalgama. }\end{array}$ & $\begin{array}{c}80 \% \\
(8)\end{array}$ & $\begin{array}{l}0 \% \\
(0)\end{array}$ & $\begin{array}{c}80 \% \\
(8)\end{array}$ & $\begin{array}{l}0 \% \\
(0)\end{array}$ \\
\hline
\end{tabular}

* (A) Alfa= Ausencia de caries secundaria. * (C) Charlie= Presencia de caries secundaria. 
dientes restaurados con amalgama utilizando barniz $^{18,19}$ Mathew y colab. ${ }^{14}$ sostienen que, además de la resistencia a la fractura, la disminución de la microfiltración (por mantenimiento de la integridad marginal) y la resistencia al desarrollo de la caries en el margen, debido a la liberación de fluoruro de lonómero Vítreo, sugieren una ventaja definitiva para el concepto de restauraciones de piezas dentarias posteriores con gran destrucción de paredes cavitarias Esto coincide con nuestros resultados, los cuales han demostrado ausencia de brechas en la interfase diente/restauración en el $100 \%$ de las piezas dentarias obturadas con lonómero Vítreo modificado con Resina/ Amalgama y en un $80 \%$ de los casos del grupo obturado con lonómero Vítreo Convencional/ Amalgama. Registrándose además la ausencia de caries secundaria en la totalidad de los casos de ambos grupos experimentales.

\section{Conclusión}

Las evaluaciones clínicas de las restauraciones, a los 3 y 6 meses, demostraron que las piezas dentarias restauradas con lonómero Vítreo modificado con Resina/Amalgama presentaron un mayor porcentaje de éxito que el grupo restaurado con lonómero Vítreo Convencional/ Amalgama, observándose además la ausencia de caries secundaria en la totalidad de los casos examinados.

Se propone la realización de investigaciones clínicas con controles a largo plazo y fin de corroborar los resultados obtenidos en este estudio.

\section{Bibliografía}

I. Mitchell RJ, Koike M, Okabe T. Posterior amalgam restorations-usage, regulation, and longevity. Dent Clin North Am. 2007; 5 I (3): 573 - 89.

2. Opdam NJ, Bronkhorst EM, Loomans BA, Huysmans MC. 12-year survival of composite vs. amalgam restorations. J Dent Res. 2010; 89 (10): 1063 $-67$.

3. Opdam NJ, Bronkhorst EM, Roeters JM, Loomans BA. A retrospective clinical study on longevity of posterior composite and amalgam restorations. Dent Mater. 2007; 23 (I): 2 - 8.

4. Heintze SD, Rousson V. Clinical effectiveness of direct class II restorations - a meta-analysis. J Adhes Dent. 20I2; 14 (5): 407- 31.

5. Antony K, Genser D, Hiebinger C, Windisch F. Longevity of dental amalgam in comparison to composite materials. GMS Health Technol Assess. 2008; 4: Docl 2.

6. Gordan VV, Riley JL 3rd, Blaser PK, Mondragon E, Garvan CW, Mjör IA. Alternative treatments to replacement of defective amalgam restorations: results of a seven-year clinical study. J Am Dent Assoc. 201 I; 142 (7): 842 - 49.

7. Bernardo M, Luis H, Martin MD, Leroux BG, Rue $T$, Leitão J, DeRouen TA. Survival and reasons for failure of amalgam versus composite posterior restorations placed in a randomized clinical trial. J Am Dent Assoc. 2007; 138 (6): 775 - 83.

8. Hoshi AT, Sliva SM, Pavarini A. In vitro evaluation of the marginal microleakage of amalgam restorations associated with dentin adhesive, glass ionomer cement and cavity varnish by means of different evaluation methods. J Appl Oral Sci 2005; 13: 10 - 4 .

9. Mathew BP, Hegde MN, Hegde P. Fracture resistance of molars with bonded class II Amalgam restorations -an in- vitro study. J Conserv Dent 2006;9:43.

10. van Duinen RN. New approach for handling glass ionomers in restorative dentistry. Refuat Hapeh Vehashinayim. 20I I; 28 (3): 8 - 13, 68.

II. Özcan M, Mese A. Adhesion of conventional and simplified resin-based luting cements to superficial and deep dentin. Clin Oral Investig. 2012; 16 (4): 1081 - 88.

12. Tyas MJ. Desempeño clínico de los cementos de ionómero Vitreos. J Minim Interv Dent 2008; I (2): 88 - 94.

13. Rasheed AA. Effect of bonding amalgam on the reinforcement of teeth. J Prosthet Dent 2005; 93: $5 \mathrm{I}-5$.

14. Mathew VB, Ramachandran S, Indira R, Shankar $P$. Comparison of the push-out shear bond strength of four types of glass ionomers when used to bond amalgam: An in vitro study. J Conserv Dent 20II; 14: 337 - 40.

15. Ryge, G. Evaluating the clinical quality of restorations. J. Am Dent Assoc. 1972; (87): 369 - 377.

16. Ryge G, Jendresen MD, Glantz PO, Mjör I. Standardization of Clinical Investigators for Studies of Restorative Materials. Swed Dent J. 198I; 5 (5-6): 235 - 39.

17. Croll TP, Bar- Zion Y, Segura A, Donly K. Clinical Performance of resin- modified glass ionomer cement restorations in primary teeth. A retrospective evaluation. JADA. 200।; 132: III0 - I5.

18. Chen RS, Liu CC, Cheng MR, Lin C. Bonded amalgam restorations: Using a Glass lonomer as an adhesive liner. Oper Dent 2000;25: 4II - 17.

19. Setcos JC, Staninec M, Wilson NH. Bonding of amalgam restorations: Existing knowledge and future prospects. Oper Dent 2000; 25: I2I - 29. 